\title{
Roton confinement in trapped dipolar Bose-Einstein condensates
}

\author{
M. Jona-Lasinio, K. Eakomy and L. Santos \\ Institut für Theoretische Physik, Leibniz Universität, 30167 Hannover, Germany
}

(Dated: June 13, 2021)

\begin{abstract}
Roton excitations constitute a key feature of dipolar gases, connecting these gases with superfluid helium. We show that the density dependence of the roton minimum results in a spatial roton confinement, particularly relevant in pancake dipolar condensates with large aspect ratios. We show that roton confinement plays a crucial role in the dynamics after roton instability, and that arresting the instability may create a trapped roton gas revealed by confined density modulations. We discuss the local susceptibility against density perturbations, which we illustrate for the case of vortices. Roton confinement is expected to play a key role in experiments.
\end{abstract}

PACS numbers: 03.75.Kk 05.30.Jp and 67.85.-d

\section{INTRODUCTION}

Dipolar gases have attracted growing attention in recent years. Quantum degenerate gases of magnetic atoms as chromium [1], dysprosium [2], and erbium [3] have already been realized. Moreover, the preparation of heteronuclear molecules in their ro-vibrational ground state [4] opens the path for the creation of a degenerate gas of polar molecules, a goal currently pursued by various groups worldwide [5]. Rydberg atoms provide yet another possible realization of a highly polar gas 6 .

The rich physics of dipolar gases arises from dipoledipole interactions (DDIs) 7]. Dipolar Bose-Einstein condensates (dBECs) feature a geometry-dependent stability [8] and a peculiar dispersion of the elementary excitations. Nonpolar BECs present the usual Bogoliubov spectrum, with a linear (phonon) dispersion at low momenta and a quadratic dispersion at large momenta 9]. Under proper conditions, dBECs present a dispersion minimum at intermediate momenta [10, 11] resembling the roton minimum of superfluid He [12.

Roton excitations are crucial in He, reducing the critical superfluid velocity [13] and leading to density modulations at defects [14 18. Remarkably, similar effects have also been predicted in dBECs 19]22. Moreover, the roton minimum is crucial for the stability of a dBEC. When the minimum reaches zero energy, the dBEC becomes unstable against finite-momentum excitations (roton instability) [10, 11, 23], which differs fundamentally from the usual phonon instability.

Roton properties in He may be controlled by means of pressure 24. Similarly, the roton minimum in dBECs depends on interactions and therefore on density. In this paper we demonstrate that this density dependence leads to a spatial roton confinement in trapped dBECs. Roton confinement has been hinted in recent numerical calculations 21, 25 and resembles that of rotons at vortex lines in $\mathrm{He}[26$. We show that roton confinement is crucial to understand the roton instability in pancake traps. We also discuss how arresting the instability may create a trapped roton gas. We finally analyze other consequences of the roton confinement, such as local susceptibility against density perturbations.

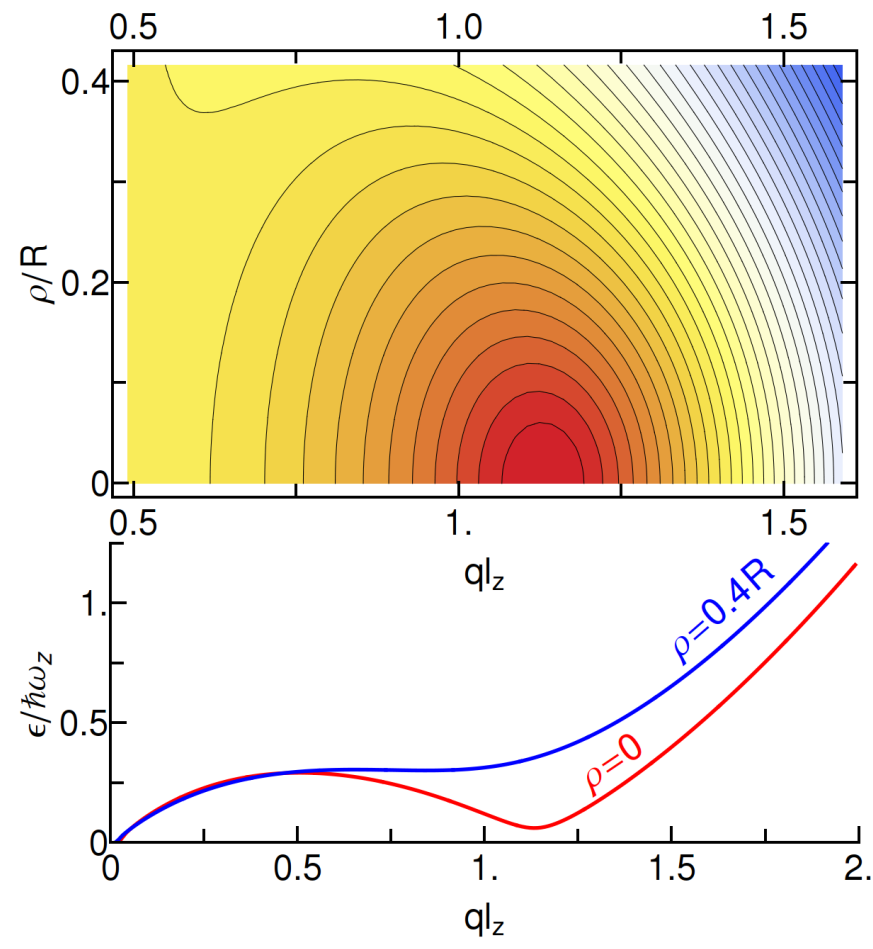

FIG. 1: (Color online) (Top) Local spectrum of a BEC of $2 \times 10^{5}$ Er atoms $\omega_{z}=2 \pi \times 1 \mathrm{kHz}$ and $\lambda=40$. Note a minimum (dark red region in the top panel) in both space and momentum. (Bottom) Change of the roton minimum for two different radial positions.

The paper is organized as follows. In Sec. II we present the system under consideration. In Sec. III we briefly review the main results of Ref. [10. The key idea of local spectrum is introduced in Sec.[IV] In Sec. V V we obtain the wave functions of the confined rotonlike excitations. Section VI is devoted to the key role played by roton confinement in the dynamics following roton instability, whereas Sec. VII analyzes the main features of the localized roton instability in time-of-flight pictures. In Sec. VIII we comment on the realization of a confined roton gas by means of a temporal destabilization of the condensate. In Sec. IX] we discuss the local susceptibility associated 
with the idea of local spectrum, focusing on the particular case of vortex lattices. Finally we summarize our conclusions in Sec. X.

\section{MODEL}

We consider a dBEC of $N$ bosons of mass $m$ and (electric or magnetic) dipole moment $d$ oriented along $z$. The dBEC is in a pancake harmonic trap $V_{t}(\mathbf{r})$ of frequencies $\omega$ in the $x y$ plane and $\omega_{z}=\lambda \omega$ along $z$, with $\lambda \gg 1$. The dBEC wave function $\phi(\mathbf{r}, t)$ obeys the nonlocal GrossPitaevskii equation (GPE) [7,

$$
\begin{aligned}
i \hbar \frac{\partial}{\partial t} \phi(\mathbf{r}, t)= & {\left[-\frac{\hbar^{2} \nabla^{2}}{2 m}+V_{t}(\mathbf{r})+g|\phi(\mathbf{r}, t)|^{2}\right] \phi(\mathbf{r}, t) } \\
& +\int d^{3} r^{\prime} V_{d d}\left(\mathbf{r}-\mathbf{r}^{\prime}\right)\left|\phi\left(\mathbf{r}^{\prime}, t\right)\right|^{2} \phi(\mathbf{r}, t),
\end{aligned}
$$

where $g=4 \pi \hbar^{2} a N / m$ characterizes the short-range interactions, $a$ is the $s$-wave scattering length, $V_{d d}(\mathbf{r})=$ $\frac{N d^{2}}{r^{3}}\left(1-3 \cos ^{2} \theta\right)$ is the DDI potential, $\theta$ is the angle between $\mathbf{r}$ and the $z$ axis, and $\int d^{3} r|\phi(\mathbf{r}, t)|^{2}=1$.

\section{HOMOGENEOUS CASE}

We first consider $\omega=0$, briefly summarizing the results of Ref. [10] (for more details, see the Appendix A. The ground-state wavefunction is $\phi_{0}(z) \exp (-i \mu t / \hbar)$, where $\mu$ is the chemical potential and $\phi_{0}(z)$ fulfills a one-dimensional (1D) local GPE with a regularized coupling constant $g+g_{d}$, with $g_{d}=8 \pi N d^{2} / 3$. Assuming a transverse Thomas-Fermi (TF) profile $\phi_{0}(z)=\sqrt{n_{0}\left(1-z^{2} / L^{2}\right)}$, with $n_{0}$ the peak density, one obtains $\mu=\left(g+g_{d}\right) n_{0}$. Excitations of energy $\epsilon$ are evaluated by substituting $\phi(\boldsymbol{\rho}, z, t)=$ $e^{-i \mu t / \hbar}\left[\phi_{0}(z)+u(\boldsymbol{\rho}, z) e^{-i \epsilon t / \hbar}-v(\boldsymbol{\rho}, z)^{*} e^{i \epsilon t / \hbar}\right] \quad[$ with $\boldsymbol{\rho} \equiv(x, y)]$ into Eq. (1), and keeping only linear terms in $u$ and $v$. The excitations have a defined in-plane momentum q, and hence $f_{ \pm}(\boldsymbol{\rho}, z) \equiv[u(\boldsymbol{\rho}, z) \pm v(\boldsymbol{\rho}, z)]=$ $f_{ \pm}(z) e^{i \mathbf{q} \cdot \boldsymbol{\rho}}$. The lowest eigenenergy obtained from the Bogoliubov de Gennes (BdG) equations for each q builds the dispersion $\epsilon(q)$. Interestingly, $\epsilon(q)$ may present a rotonlike minimum at intermediate $q$. Assuming $q L \gg 1$ and $\mu E(q)|\beta-2| /(\beta+1) \lesssim \hbar^{2} \omega_{z}^{2}$ (with $\beta \equiv g_{d} / g$ and $\left.E(q) \equiv \hbar^{2} q^{2} / 2 m\right)$ we obtain an approximate expression of the dispersion, $\epsilon_{h}^{2}(q, \mu)=E(q)^{2}-G(\beta) E(q) \mu+\hbar^{2} \omega_{z}^{2}$, with $G(\beta) \equiv \frac{(\beta-2)(5 \beta+2)}{3(1+\beta)(2 \beta+1)}$, as well as the associated eigenstate, $f_{+}(z) \simeq \phi_{0}(z)$. The expression $\epsilon_{h}(q, \mu)$ agrees well with the numerical $\epsilon(q)$ and shows that the roton depth depends explicitly on $\mu$ and hence on density.

\section{LOCAL SPECTRUM}

Interesting insights about the roton physics for $\omega>0$ are provided by the concept of local spec- trum $\epsilon(q, \rho)$, which we introduce here. We compute from Eq. (1) the ground-state $n_{0}(\mathbf{r})=\left|\phi_{0}(\mathbf{r})\right|^{2}$, which in the $\mathrm{TF}$ regime is approximated by $n_{0}(\mathbf{r})=$ $\tilde{n}_{0}\left(1-\rho^{2} / R^{2}-z^{2} / L^{2}\right)$ [27. We obtain for each $\rho$ the $z$ profile $n_{0}^{1 \mathrm{D}}(z)=n_{0}(\mathbf{r}) / \int d z n_{0}(\mathbf{r})$ and the local chemical potential $\mu_{l}(\rho)$. Solving the corresponding 1D BdG equations [10] we obtain $\epsilon_{h}\left(q, \mu_{l}(\rho)\right)$, which approximates the local spectrum $\epsilon(q, \rho)$ consistently with the LDA. The local chemical potential $\mu_{l}(\rho)$ decreases quadratically with $\rho$, and hence at the trap center $(\rho=0)$ the roton energy is lowest: $\epsilon_{r} / \hbar \omega_{z} \equiv \epsilon(q=$ $\left.q_{r}, \rho=0\right) / \hbar \omega_{z} \simeq \sqrt{1-\left(G(\beta) \mu_{l}(0) / 2 \hbar \omega_{z}\right)^{2}}$. The local spectrum presents a minimum both in momentum, at $q_{r} l_{z} \simeq \sqrt{G(\beta) \mu_{l}(0) / \hbar \omega_{z}}$ (note that $q_{r} l_{z} \sim 1$, with $l_{z} \equiv \sqrt{\hbar / m \omega_{z}}$, see [10), and in space, at $\rho=0$ (Fig. 1). For larger $\rho$ the minimum becomes shallower and eventually disappears. Hence, remarkably, the inhomogenous BEC density results in a spatial roton confinement. Around the minimum,

$$
\epsilon(q, \rho) \simeq \epsilon_{r}+\frac{\hbar^{2}\left(q-q_{r}\right)^{2}}{2 m_{*}}+\frac{1}{2} m_{*} \omega_{*}^{2} \rho^{2},
$$

where the effective roton mass $m_{*} \equiv m \sqrt{1 /\left(q_{r} l_{z}\right)^{4}-1 / 4}$ and the effective harmonic frequency $\omega_{*} \equiv \omega_{z} \frac{m q_{r} l_{z}^{2}}{m_{*} R \sqrt{2}}$ define the roton localization length $l_{*} \equiv \sqrt{\hbar / m_{*} \omega_{*}}=$ $2^{1 / 4}\left(R / q_{r}\right)^{1 / 2}$. Note that $l_{*} / R \sim \sqrt{l_{z} / R}$, and hence $l_{*} \ll R$ for $\lambda \gg 1$. Moreover, $q_{r} l_{*} \sim \sqrt{R / l_{z}} \gg 1$ if $\lambda \gg 1$, which justifies the use of LDA above.

\section{LOCALIZED ROTON WAVEFUNCTION}

We now calculate the localized roton wave functions using the LDA and the formalism of Ref. [10] (for more details, see the Appendix A. Assuming $l_{*} \ll R$, we approximate $f_{+}(\boldsymbol{\rho}, z) \simeq F(\boldsymbol{\rho}) \phi_{0}(\boldsymbol{\rho}, z)$, where $F(\boldsymbol{\rho})$ has a narrow momentum distribution $\tilde{F}(\mathbf{q})$ centered around $q_{r}$ with a width $\delta q \propto 1 / l_{*} \ll q_{r}$. The finite width of $\tilde{F}(\mathbf{q})$ must be now considered and as a result $\tilde{F}(\mathbf{q})$ fulfills the eigenvalue equation $\epsilon^{2} \tilde{F}(\mathbf{q}) \simeq\left[\epsilon_{r}+\hat{H}\right]^{2} \tilde{F}(\mathbf{q})$, with $\hat{H} \equiv \frac{\hbar^{2}}{2 m_{*}}\left(q-q_{r}\right)^{2}-\frac{1}{2} m_{*} \omega_{*}^{2} \nabla_{\mathbf{q}}^{2}$. Interestingly, $\hat{H}$ resembles the Hamiltonian of a trapped BEC in the presence of spin-orbit coupling [28, 29], where the Rashba-like dispersion $\sim\left(q-q_{r}\right)^{2}$ acts as a ringlike potential in $\mathbf{q}$ space. The eigenfunctions of $\hat{H}, \eta_{n, s}(q) e^{i s \varphi} / \sqrt{q}$, fulfill

$$
\left[\frac{E_{n, s}}{\hbar \omega_{*}}-\frac{s^{2}-\frac{1}{4}}{2\left(q l_{*}\right)^{2}}\right] \eta_{n, s}=\left[-\frac{1}{2 l_{*}^{2}} \frac{d^{2}}{d q^{2}}+\frac{l_{*}^{2}}{2}\left(q-q_{r}\right)^{2}\right] \eta_{n, s} .
$$

For $q_{r} l_{*} \gg 1$, we expand around $q \simeq q_{r}$, obtaining the eigenenergies $E_{n, s} / \hbar \omega_{*} \simeq\left(s^{2}-1 / 4\right) /\left(2\left(q_{r} l_{*}\right)^{2}\right)+n+$ $1 / 2$, characterized by the angular momentum $s$ around the Rashba-like ring, and the radial harmonic excitations, $n$, with frequency $\omega_{*}$. The lowest roton states 

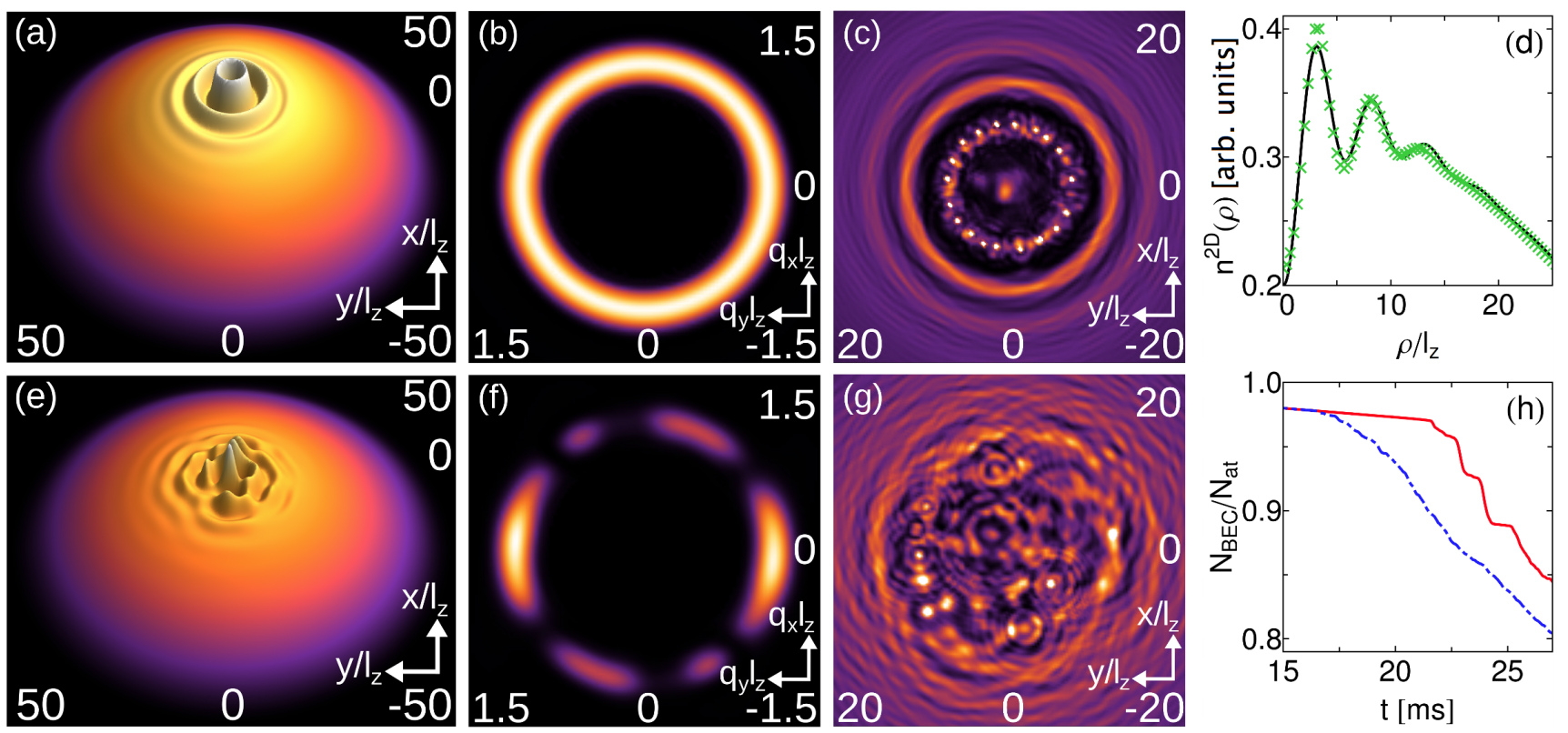

FIG. 2: (Color online) Roton instability for $10^{5}$ Er atoms, with $\omega_{z}=2 \pi \times 450 \mathrm{~Hz}\left(l_{z} \simeq 0.3 \mu \mathrm{m}\right), \lambda=30, a_{i}=8.49 a_{0}>$ $a_{c}=8.48 a_{0}$ and $a_{f}=0$ (see text). (a) Column density $n^{2 D}(\boldsymbol{\rho})=\int n(\mathbf{r}) d z$ showing concentric rings $(s=0)$ formed after $t=19 \mathrm{~ms}$ for small initial fluctuations $\left(\xi=10^{-10}\right.$; see text). (e) Modulational instability after $t=15.5 \mathrm{~ms}$ consisting of several $s$ states for large initial fluctuations $\left(\xi=10^{-6}\right.$; see text). (b) and (f): momentum distribution of (a) and (e) respectively (we have suppressed the large peak at $q=\sqrt{q_{x}^{2}+q_{y}^{2}}=0$ ). (c) and (g): post-collapse dynamics after $t=23$ $\mathrm{ms}$ for (a) and $t=19.5 \mathrm{~ms}$ for (e), respectively. (d) Radial cut of (a) (green crosses) and theoretical column density of the form $n^{2 D}(\boldsymbol{\rho}) \propto\left(1-\rho^{2} / R^{2}\right)^{3 / 2}\left(1+A J_{0}\left(q_{r} \rho\right) e^{-\rho^{2} / 2 l_{*}^{2}}\right.$ ) (solid black line), with $R \simeq 51 l_{z}, A \simeq-0.4, q_{r} l_{z} \simeq 1.25$, and $l_{*}=2^{1 / 4}\left(R / q_{r}\right)^{1 / 2} \simeq 7.6 l_{z}$. The value of $q_{r}$ is calculated using the local spectrum picture. (h) Remnant atoms for the cases (a) (solid red line) and (e) (dashed blue line).

have $n=0$, being in real space of the form $\psi_{s}(\boldsymbol{\rho}) \sim$ $e^{i s \varphi} e^{-\rho^{2} / 2 l_{*}^{2}} J_{s}\left(q_{r} \rho\right)$, with $J_{s}$ the Bessel function.

\section{LOCAL ROTON INSTABILITY}

The localized states $\psi_{s}$ are crucial for the dynamics following roton instability [30. We consider a stable BEC prepared with an initial scattering length $a_{i}>a_{c}$. The critical $a_{c}$ for the onset of instability depends nontrivially on $\lambda$ and $g_{d}$ [7]; for the parameters of Fig. 2 it is $a_{c}=8.48 a_{0}$, with $a_{0}$ the Bohr radius. We are interested in the instability dynamics after a sudden quench to $a_{f}<a_{c}$. Unstable modes lead to a modulational instability characterized by a growing density perturbation

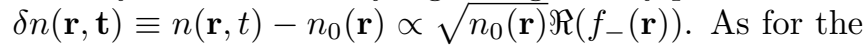
$\omega=0$ case, $f_{-} \simeq\left(E\left(q_{r}\right) / \epsilon\right) f_{+} \propto \sqrt{n_{0}(\mathbf{r})} F(\boldsymbol{\rho})$. Hence the most unstable roton modes result in a localized modulation at the trap center, $\delta n(\mathbf{r}) / n_{0}(\mathbf{r}) \propto \Re\left(\psi_{s}(\boldsymbol{\rho})\right)$.

The most unstable mode is $\psi_{0}(\boldsymbol{\rho})$, but other modes may contribute to the instability due to the small energy difference between low- $s$ levels $\left[\sim \hbar \omega_{*} /\left(q_{r} l_{*}\right)^{2}\right]$. As a nontrivial consequence of that, the density pattern that develops after the quench is influenced by the initial population of the excitations exponentially amplified during the destabilization. We mimic this dependence by considering a small initial seeding $\phi(\mathbf{r}, t=0)=\phi_{0}(\mathbf{r}) e^{i \chi(\mathbf{r})}$, where $\phi_{0}$ is the ground state calculated for $a_{i}$, and $|\chi(\mathbf{r})| / \pi<\xi$ is a random phase sampled from a homogeneous uniform distribution with a variable amplitude $\xi$. Although this allows us to discuss the possible collapse scenarios, the actual amplitude of the initial fluctuations depends on $a_{i}$ and on the temperature $T$, and its analysis lies beyond the scope of this paper [31.

Figures 2 show the results of our simulations of Eq. (1) for an erbium dBEC [32. For a small initial population of the unstable modes, the modulation instability proceeds at a sufficiently slow pace and $\psi_{0}(\boldsymbol{\rho})$ dominates. As a result, a localized pattern of concentric rings develops [Fig. 2(a)], in excellent agreement with $\delta n(\mathbf{r}) / n_{0}(\mathbf{r}) \propto \psi_{0}(\boldsymbol{\rho})$; see Fig. 2(d) [33]. The corresponding momentum distribution presents a Rashba-like ring [Fig. 2(b)] 34. In contrast, for larger initial fluctuations the pattern growth is too fast to select $\psi_{0}$ only, and the created density pattern results from a (shot-toshot dependent) linear combination of different $\psi_{s}$, being characterized by a superposition of eccentric collapse centers [Fig. 2(e)]. The corresponding momentum distribution still presents a ringlike structure but with an azimuthal modulation arising from the linear combination of various $\psi_{s}$ [Fig. 2(f)].

The global (phononlike) collapse studied in chromium and erbium dBECs 3, 35, results in large atom losses and in a $d$-wave pattern in time-of-flight (TOF) pictures. 

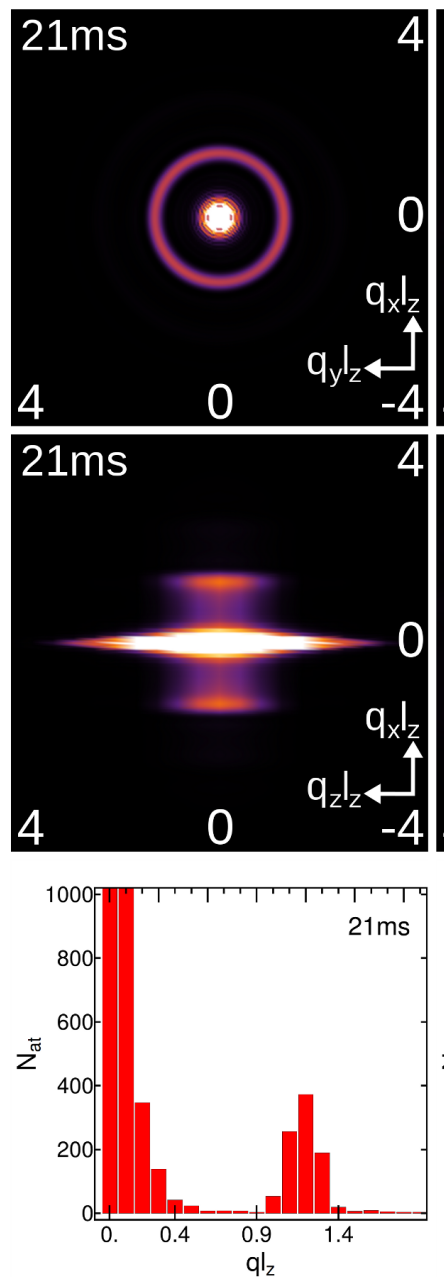
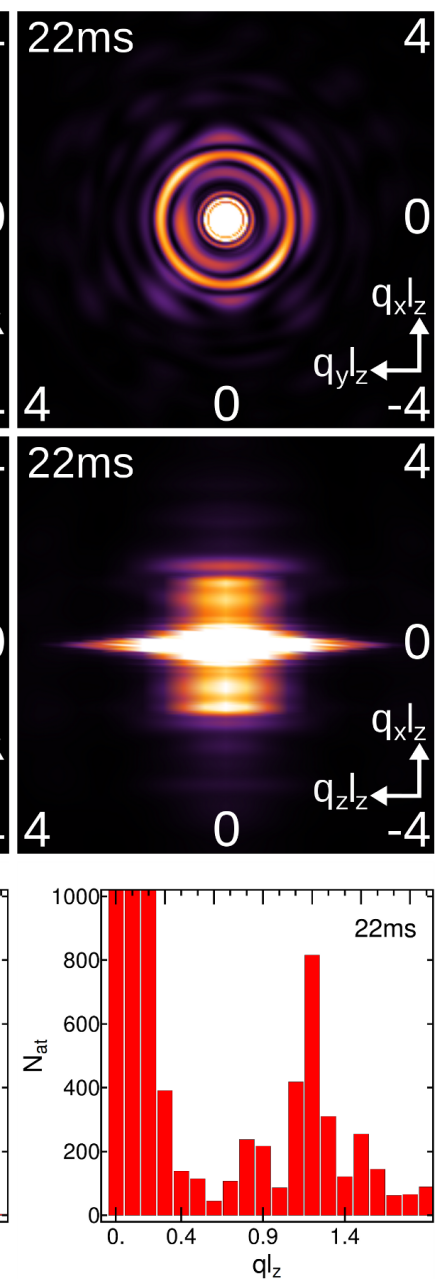
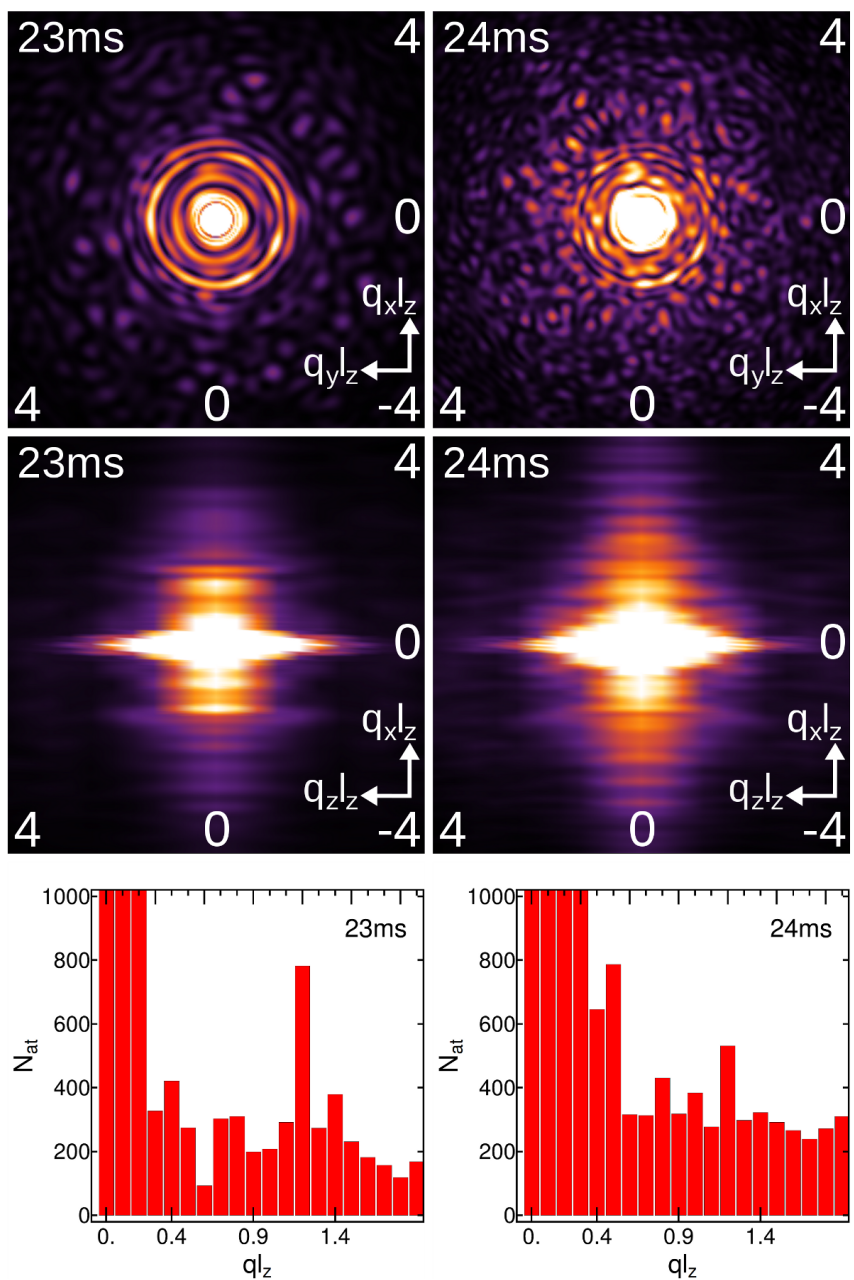

FIG. 3: (Color online) Momentum distribution for different times for the same case depicted in Fig. 2(a), as it would be revealed by a TOF experiment. In the top panel we show the $q_{x}, q_{y}$ momentum distribution (integrated over $q_{z}$ ) and in the middle panel the $q_{x}, q_{z}$ momentum distribution (integrated over $q_{y}$ ). The bottom panel shows the integrated population at different intervals of the radial momentum $q=\sqrt{q_{x}^{2}+q_{y}^{2}}$.

Remarkably, the roton instability discussed above leads to a very different collapse dynamics. Three-body losses become crucial in the collapse dynamics and we included them by adding $-i \hbar \frac{L_{3}}{2} N^{2}|\phi(\mathbf{r}, t)|^{4} \phi(\mathbf{r}, t)[35$, to Eq. (1), with a loss rate $L_{3}=10^{-28} \mathrm{~cm}^{-6} \mathrm{~s}^{-1}$. The concentric rings appearing in Fig. 2(a) eventually undergo a sequential collapse and azimuthal instability, starting from the inner (denser) ones towards the outer ones [Fig. 2(c)] leading to a step-like atom number decrease [Fig. 2(h)]. The superimposed eccentric collapse centers appearing in Fig. 2(e) lead to a complex post-collapse behavior with characteristic mutually interfering jets expelled out of each local collapse center [Fig. 2(g)]. In this case the atom decrease is smooth [Fig. 2(h)].

\section{TIME-OF-FLIGHT PICTURES}

Although the most straightforward way of studying roton confinement is of course given by in situ measure- ments [36, TOF pictures are expected to show a clear difference as well, when compared to the cloverleaf pattern characteristic of the phonon collapse (as observed in chromium [35] and, more recently, in erbium [3]). More specifically, TOF pictures may reveal clear traces of the Rashba-like ring characterizing the localized rotonlike excitations.

Due to the fast expansion after release, TOF pictures are expected to reproduce well the in situ momentum distribution at the time of releasing. We have calculated the momentum distribution of the condensate at different stages during the instability dynamics. Our results for the same case studied in Fig. 2(a) are depicted in Fig. 3, where we show both the column density on the $q_{x}-q_{y}$ plane (i.e. integrated along $q_{z}$ ) and that on the $q_{x}-q_{z}$ plane (i.e. integrated along $q_{y}$ ). We show in particular the results obtained during the first stages of the collapse dynamics. For the parameters of Fig. 2(a), the Rashbalike ring is clearly visible during the first stages of the collapse, being destroyed later on due to the subsequent 


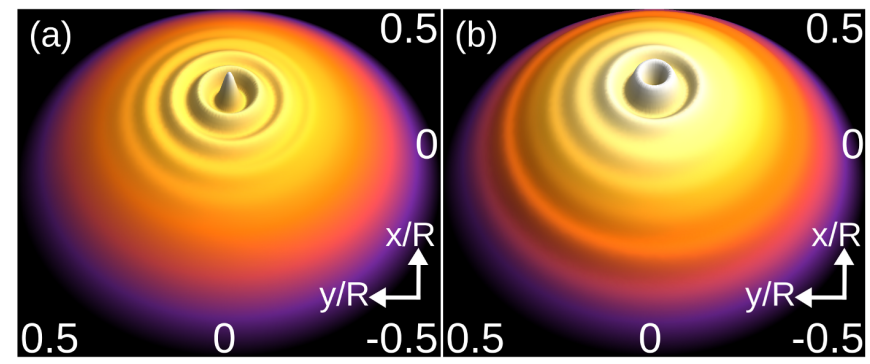

FIG. 4: (Color online) Same parameters as in Fig. 2(a) but arresting the collapse after $16 \mathrm{~ms}$ with a quench of $a$ up to $8.55 a_{0}>a_{c}$. Snapshots of the central region right after the quench (a) and $80 \mathrm{~ms}$ later (b). Note a clear density modulation confined at the trap center.

collapse of the spatial ringlike density modulations. As a result the ring TOF pattern is washed out. In later stages TOF pictures are characterized by the appearance of large momentum excitations on the $x y$ plane (induced by the collapse of the local rings) which becomes visible in the $q_{x}-q_{z}$ distribution as pronounced jets along $x$.

The low-momentum peak always appears saturated in our pictures. It was eliminated from Figs. 2(b) and $2(\mathrm{~g})$, since we were only interested in showing the Rashba-like feature in momentum space. The large low-momentum peak is the result of two effects. The first one is purely geometric. The $q=0$ peak is always relatively strong, since it is not spread in the angular variable $\varphi$ (as it is the ring feature discussed above). The second reason is more physical, and it is linked with the local nature of the collapse. Since only a small fraction of the atoms is actually participating in the modulational instability and in the subsequent collapse, a large fraction of atoms remains at low momentum. Note that the low momentum peak also broadens due to the generation of low energy phonons during the collapse. As a result, the low-momentum peak dominates the TOF pictures. This is in itself a clear difference with respect to the phonon collapse, where the initial low-momentum peak transforms into a cloverleaf pattern due to the global nature of the collapse.

However, although the relative number of particles produced in the Rashba-like ring may be small, the actual absolute number of particles may be certainly sizable. In Fig. 3 (bottom panel) we depict at various times the number of particles at different radial momenta. This plot shows that a significant number of particles is found in the ring before the ring feature is eventually washed out (up to 1500 particles at $21.5 \mathrm{~ms}$ ).

\section{CONFINED ROTON GAS}

Interestingly, the roton instability may be used to create a confined roton gas. The density pattern obtained after quenching down to $a_{f}<a_{c}$ may be interpreted as the growth of the roton population. At the initial stage of the instability, the roton population is small compared

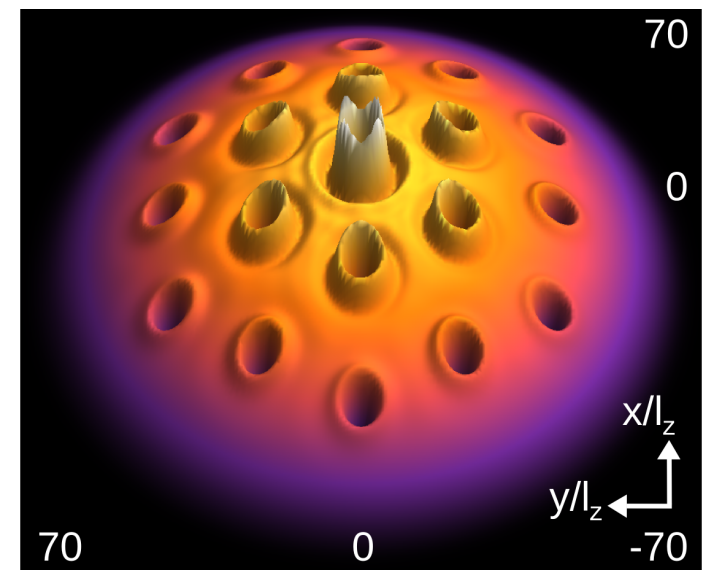

FIG. 5: (Color online) Vortex lattice for a BEC of $N=10^{5}$ Er atoms at the threshold of the roton instability. Parameters as in Fig. 1 with a rotational frequency $0.3 \omega$.

to $N$ and we may neglect condensate depletion or rotonroton interactions. Once the roton gas is populated, we stabilize it by quenching up to $a>a_{c}$. Since $m_{*}$ and $\omega_{*}$ do not vary significantly around the instability threshold, the created rotons (density patterns) remain confined at the trap center after the quench, as shown in Fig. 4.

\section{LOCAL SUSCEPTIBILITY}

A deep roton minimum induces close to a perturbation a large susceptibility against the formation of density modulations with the roton wavelength. This well-known effect in He [14 18] is also relevant for dBECs [20]. The local spectrum picture implies a spatially dependent susceptibility, which we illustrate for the case of vortices [37. Vortex cores present a craterlike shape in the case of a deep roton minimum [19, 20] that disappears when the minimum is shallow or absent. Therefore, vortices at different positions in a trapped BEC present a different core profile. This is shown for a vortex lattice in Fig. 5 , where we depict the ground state of an erbium BEC rotating around the $z$ axis with an angular frequency $\Omega=0.3 \omega$. Note that core modulations at the trap center disappear for vortices at the boundary.

\section{CONCLUSIONS}

In summary, we have shown that an inhomogeneous trapping in pancake dBECs with large aspect ratios leads to a spatial roton confinement which is crucial to understanding the roton instability. The roton dispersion has not yet been observed experimentally, being currently a major goal pursued by several groups. Roton confinement is expected to play a key role in these experiments, since harmonic traps are typically employed and large aspect ratios are needed to study the roton dis- 
persion. In addition to the local susceptibility discussed above 37, roton confinement should be carefully considered when measuring the critical superfluid velocity, performing Bragg scattering [38, or analyzing finite temperature physics, which may be very interesting since the thermal roton cloud is expected to localize at the center of the trap.

\section{Acknowledgments}

We acknowledge funding by the German-Israeli Foundation, and the DFG (Grant No. SA1031/6 and Excellenzcluster QUEST).

\section{Appendix A: Derivation of the localized roton wave functions}

\section{Homogeneous $x y$ case $(\omega=0)$}

We briefly summarize in this section the main formalism developed in Ref. 10. For $\omega=0$, the ground state is of the form $\phi_{0}(z) e^{-i \mu t / \hbar}$, where $\mu$ is the chemical potential and

$$
\left\{-\frac{\hbar^{2}}{2 m} \nabla^{2}+\frac{m}{2} \omega_{z}^{2} z^{2}+\left(g+g_{d}\right)\left|\phi_{0}(z)\right|^{2}-\mu\right\} \phi_{0}(z)=0 .
$$

We assume $\left(g+g_{d}\right)>0$. For $\mu \gg \hbar \omega_{z}$ the condensate presents a TF density profile, $\left|\phi_{0}(z)\right|^{2}=n_{0}\left(1-z^{2} / L^{2}\right)$, with $n_{0}=\mu /\left(g+g_{d}\right)$ being the maximum density, and $L=\sqrt{2 \mu / m \omega_{z}^{2}}$ the TF radius.

Excitations of energy $\epsilon$ are evaluated by substituting $\phi(\boldsymbol{\rho}, z, t)=$ $e^{-i \mu t / \hbar}\left[\phi_{0}(z)+u(\boldsymbol{\rho}, z) e^{-i \epsilon t / \hbar}-v(\boldsymbol{\rho}, z)^{*} e^{i \epsilon t / \hbar}\right] \quad[$ with $\boldsymbol{\rho} \equiv(x, y)$ ] into the 3D nonlocal GPE [Eq. (1) of our Letter], and keeping only linear terms in the Bogoliubov amplitudes $u$ and $v$. For $\omega=0$, the excitations have a defined in-plane momentum $\mathbf{q}$, and we may write $f_{ \pm}(\boldsymbol{\rho}, z) \equiv[u(\boldsymbol{\rho}, z) \pm v(\boldsymbol{\rho}, z)]=f_{ \pm}(z) e^{i \mathbf{q} \cdot \boldsymbol{\rho}}$. The BdG equations read

$$
\begin{aligned}
\epsilon f_{-} & =\frac{\hbar^{2}}{2 m}\left[-\frac{d^{2}}{d z^{2}}+q^{2}+\frac{\nabla^{2} \phi_{0}}{\phi_{0}}\right] f_{+} \equiv H_{k i n} f_{+}, \\
\epsilon f_{+} & =H_{k i n} f_{-}+H_{\text {int }}\left[f_{-}\right],
\end{aligned}
$$

with

$$
\begin{aligned}
H_{\text {int }}\left[f_{-}\right] & =2\left(g_{d}+g\right) f_{-}(z)\left|\phi_{0}(z)\right|^{2}-(3 / 2) g_{d} q \phi_{0}(z) \\
& \times \int_{-\infty}^{\infty} d z^{\prime} f_{-}\left(z^{\prime}\right) \phi_{0}\left(z^{\prime}\right) \exp \left(-q\left|z-z^{\prime}\right|\right) .
\end{aligned}
$$

For each $q$ we calculate the eigenenergies. The lowest one, $\epsilon(q)$ builds the dispersion law.

The most interesting behavior occurs for $q L \gg 1$. We may now introduce $f_{+}(z)=W(z) \phi_{0}(z)$. Expressing $f_{-}$ through $W$ from Eq. A2, we substitute it into Eq. A3) and integrate over $d z$ in $H_{\text {int }}\left[f_{-}\right]$as the main contribution to the integral comes from a narrow range of distances $\left|z^{\prime}-z\right| \sim 1 / q$. This yields (here $\chi=z / L$ )

$$
\begin{aligned}
& \hbar^{2} \omega^{2}\left[\frac{1}{2}\left(1-\chi^{2}\right) \frac{d^{2}}{d \chi^{2}}-\left(1+\frac{3}{2(1+\beta)}\right) \chi \frac{d}{d \chi}\right] W(\chi) \\
= & -\left[\epsilon^{2}-E(q)^{2}-\frac{2 \beta-1}{1+\beta} \mu E(q)\left(1-\chi^{2}\right)-\frac{3 \hbar^{2} \omega^{2}}{2(1+\beta)}\right] W(\chi)=0,
\end{aligned}
$$

where $E(q)=\hbar^{2} q^{2} / 2 m$. Here we omitted terms of the order of $E(q) \hbar^{2} \omega^{2} / \mu$ and $\hbar^{4} \omega^{4} / \mu^{2}$, since they are small compared to either $\hbar^{2} \omega^{2}$ or $E(q)^{2}$. For each mode of the confined motion (along the $z$ direction), the solution of Eq. A5 can be written as an expansion series in Gegenbauer polynomials $C_{n}^{\lambda}(\chi)$, where $\lambda=(4+\beta) / 2(1+\beta)$, and $n \geq 0$ is an integer. For $\mu E(q)|2 \beta-1| /(1+\beta) \lesssim \hbar^{2} \omega^{2}$ the lowest solution is of the form $W(\chi) \simeq 1+\sum_{n>0} a_{n} C_{n}^{\lambda}(\chi)$, with small amplitudes $a_{n}$ of Gegenbauer polynomials of higher order, while the lowest-eigenenergy (and hence the dispersion) is given by

$$
\epsilon_{h}^{2}(q, \mu)=E(q)^{2}-G(\beta) E(q) \mu+\hbar^{2} \omega_{z}^{2},
$$

with $G(\beta) \equiv \frac{(\beta-2)(5 \beta+2)}{3(1+\beta)(2 \beta+1)}$.

\section{Trapped $x y$ case}

We consider in the following $\omega>0$, with $\lambda=\omega_{z} / \omega \gg$ 1. Due to the $x y$ confinement, we cannot employ anymore a plane wave basis for the Bogoliubov amplitudes, as we did above. However, we assume that (i) the Bogoliubov amplitudes are, in momentum space, strongly peaked around a momentum $q \gg 1 / R$ and (ii) the Bogoliubov amplitudes are localized at the center of the trap, in a region with a radius much smaller than $R$. These assumptions must be checked self-consistently, but we anticipate that they are fulfilled for a sufficiently large aspect ratio $\lambda \gg 1$. If this is the case, we may proceed as for the homogeneous case, but taking into account that (a) the chemical potential is now $\rho$ dependent, and (b) 
the Bogoliubov amplitudes are not plane waves anymore. Assuming the above conditions are met, we may write $f_{+}(\boldsymbol{\rho}, z) \simeq F(\boldsymbol{\rho}) W(x) \phi_{0}(\boldsymbol{\rho}, z)\left(\right.$ now $\left.x^{2}=\rho^{2} / R^{2}+z^{2} / L^{2}\right)$, where the function $F(\boldsymbol{\rho})$ has a narrow momentum dis- tribution $F(\mathbf{q})$ peaked at $q_{r}$ with a momentum width $\delta q \propto 1 / l_{*} \ll q_{r}$ and $\phi_{0}(\boldsymbol{\rho}, z)$ is the ground state. Taking into account (a) and (b), we rewrite Eq. A5 in the form

$$
\begin{aligned}
& \hbar^{2} \omega^{2}\left[\frac{1}{2}\left(1-x^{2}\right) \frac{d^{2}}{d x^{2}}-\left(1+\frac{3}{2(1+\beta)}\right) x \frac{d}{d x}\right] W(x) F(\boldsymbol{\rho}) \\
= & -\left[\epsilon^{2}-E(\hat{\mathbf{q}})^{2}-\frac{2 \beta-1}{1+\beta} \mu E(\hat{\mathbf{q}})\left(1-x^{2}\right)-\frac{3 \hbar^{2} \omega^{2}}{2(1+\beta)}\right] W(x) F(\boldsymbol{\rho})=0,
\end{aligned}
$$

where $\hat{\mathbf{q}} \equiv-i \boldsymbol{\nabla}$. The lowest energy eigenstates still fulfill $W(x) \simeq 1$, but now we must keep explicitly the spatial dependence of $F(\boldsymbol{\rho})$,

$$
\epsilon^{2} F(\boldsymbol{\rho})=\left[E(\hat{\mathbf{q}})^{2}-G(\beta) E(\hat{\mathbf{q}}) \mu(\rho)+\hbar^{2} \omega_{z}^{2}\right] F(\boldsymbol{\rho}) .
$$

Expanding around the roton minimum in the local spectrum, and moving to momentum space $\left(\boldsymbol{\rho}=i \boldsymbol{\nabla}_{q}\right)$ we obtain

$$
\epsilon^{2} \tilde{F}(\mathbf{q})=\left[\epsilon_{r}+\frac{\hbar^{2}\left(q-q_{r}\right)^{2}}{2 m_{*}}-\frac{1}{2} m_{*} \omega_{*}^{2} \nabla_{q}^{2}\right]^{2} \tilde{F}(\mathbf{q})
$$

where $\tilde{F}(\mathbf{q})$ is the Fourier transform of $F(\boldsymbol{\rho})$. Hence $\tilde{F}(\mathbf{q})$ are the eigenstates of the Hamiltonian $\hat{H} \equiv \frac{\hbar^{2}\left(q-q_{r}\right)^{2}}{2 m_{*}}-$ $\frac{1}{2} m_{*} \omega_{*}^{2} \nabla_{q}^{2}$, which may be calculated as discussed in Sec. V.

\section{Density modulations}

Note that density modulations are given by the $f_{-}$ amplitudes, in the form: $\delta n(\mathbf{r}, \mathbf{t}) \equiv n(\mathbf{r}, t)-n_{0}(\mathbf{r}) \propto$ $\sqrt{n_{0}(\mathbf{r})} \Re\left(f_{-}(\mathbf{r})\right)$., where $\Re$ denotes the real part. For the $\omega=0$ case, and since $W(\chi) \simeq 1$, from Eq. A2 one obtains that $f_{-} \simeq(E(q) / \epsilon) f_{+}$. For the $\omega>0$ case, since $W(x) \simeq 1$ still, and we assume that $\tilde{F}(\mathbf{q})$ is narrowly peaked around $q_{r}$, we obtain the same result for the trapped case. Hence, apart from a constant, $f_{-}(\mathbf{r}) \propto f_{+}(\mathbf{r}) \propto \psi_{0}(\mathbf{r}) F(\boldsymbol{\rho})$. As a result, $\delta n(\mathbf{r}, \mathbf{t}) \propto$ $n_{0}(\mathbf{r}) \Re(F(\boldsymbol{\rho}))$.
[1] A. Griesmaier et al., Phys. Rev. Lett. 94, 160401 (2005); Q. Beaufils, et al., Phys. Rev. A 77, 061601 (2008).

[2] M. Lu et al., Phys. Rev. Lett. 107, 190401 (2011); M. Lu, N. Q. Burdick, and B. L. Lev, Phys. Rev. Lett 108, 215301 (2012).

[3] K. Aikawa, et al., Phys. Rev. Lett. 108, 210401 (2012).

[4] K.-K. Ni, et al., Science 322, 231 (2008); M. H. G. de Miranda, et al., Nat. Phys. 7, 502 (2011); A. Chotia, et al., Phys. Rev. Lett. 108, 080405 (2012).

[5] C.-H. Wu et al., Phys. Rev. Lett. 109, 085301 (2012); T. Takekoshi et al., Phys. Rev. A 85, 032506 (2012).

[6] T. F. Gallagher and P. Pillet, in Adv. At., Mol.,Opt. Phys. 56, 161 (2008); Robert Löw et al., J. Phys. B: At. Mol. Opt. Phys. 45, 113001 (2012).

[7] See M. A. Baranov, Phys. Rep. 464, 71 (2008); T. Lahaye et al., Rep. Prog. Phys. 72, 126401 (2009); M. A. Baranov et al., Chem. Rev. 112, 5012 (2012).

[8] S. Müller et al., Phys. Rev. A 84, 053601 (2011)

[9] L. Pitaevskii and S. Stringari, Bose-Einstein condensation, Oxford Univ. Press (New York, 1993).

[10] L. Santos, G. V. Shlyapnikov, and M. Lewenstein, Phys. Rev. Lett. 90, 250403 (2003).

[11] S. Ronen, D. C. E. Bortolotti, and J. L. Bohn, Phys. Rev. Lett. 98, 030406 (2007).
[12] L. D. Landau, J. Phys. USSR 11, 91 (1947); Phys. Rev. 75, 884 (1949); R. P. Feynman, Phys. Rev. 94, 262 (1954).

[13] L. Landau, J. Phys. (Moscow) 5, 71 (1941).

[14] T. Regge, J. Low Temp. Phys. 9, 123 (1972).

[15] F. Dalfovo, Phys. Rev. B 46, 5482 (1992).

[16] Y. Pomeau and S. Rica, Phys. Rev. Lett. 71, 247 (1993).

[17] N. G. Berloff and P. H. Roberts, J. Phys. A 32, 5611 (1999).

[18] S. Villerot, B. Castaing, and L. Chevillard, J. Low Temp. Phys. 169, 1 (2012).

[19] S. Yi and H. Pu, Phys. Rev. A 73, 061602(R) (2006).

[20] R. M. Wilson, S. Ronen, J. L. Bohn, and H. Pu, Phys. Rev. Lett. 100, 245302 (2008).

[21] R. M. Wilson, S. Ronen, and J. L. Bohn, Phys. Rev. Lett. 104, 094501 (2010).

[22] H.-Y. Lu et al., Phys. Rev. A 82, 023622 (2010).

[23] R. M. Wilson, S. Ronen, and J. L. Bohn, Phys. Rev. A 80, 023614 (2009).

[24] O. W. Dietrich et al., Phys. Rev. A 5, 1377 (1972); E. C. Svensson, A. D. B. Woods, and P. Martel, Phys. Rev. Lett. 29, 1148 (1972); P. R. Roach et al., J. Low Temp. Phys. 12, 375 (1973).

[25] P. B. Blakie, D. Baillie, and R. N. Bisset, Phys. Rev. A 
86, 021604 (2012).

[26] I. Iguchi, Phys. Rev. A 6, 1087 (1972).

[27] We assume the BEC away from the narrow regions of biconcave density profiles [11.

[28] T. D. Stanescu, B. Anderson and V. Galitski, Phys. Rev. A 78, 023616 (2008).

[29] S. Sinha, R. Nath, and L. Santos, Phys. Rev. Lett. 107, 270401 (2011).

[30] Roton instability was discussed in Ref. [23]. However, roton confinement played no role there, due to the low $\lambda$ considered. However, confinement effects might be inferred from an unpublished numerical simulation (http://grizzly.colorado.edu/ bohn/ movies/collapse.htm). In our numerics, we observed localized modulational instabilities for $\lambda \gtrsim 14$.

[31] In our calculations $a_{i}$ is chosen close to $a_{c}$, but its actual value is not relevant, since we just employ the groundstate for $a=a_{i}$ and add numerical noise. However, the value of $a_{i}$ may be relevant for the actual fluctuations. If the spectrum is weakly (or not) rotonized for $a_{i}$, the initial population of the dominant unstable modes for $a=a_{f}$ (which are around the roton minimum) is negligible when $k_{B} T \ll \mu_{l}(0)$ ( $k_{B}$ is the Boltzmann constant), corresponding to small $\xi$ in Fig. 2 In contrast, the initial population of unstable modes may be significant for $k_{B} T \sim \epsilon_{r}$, if the roton depth approaches zero for $a_{i}$, corresponding to large $\xi$ in Fig. 2 .

[32] Similar results are expected for other magnetic species such as chromium and dysprosium, although the particular parameters will, of course, change.

[33] Concentric rings were observed by N. G. Parker et al., Phys. Rev. A 79, 013617 (2009). However, the ring structure was not localized and did not result from roton confinement, but from dynamically unstable phonon modes.

[34] Prior to destabilization the dBEC presents a narrow momentum distribution at $q=0$ with a width $\propto 1 / R$. We have removed the $q=0$ contribution in Figs. 2(b) and 2 (f), to highlight the ring-like pattern as well as the azimuthal structure along the ring when several $s$ states are mixed. A thorough analysis of the momentum distribution is performed in Sec. VII

[35] T. Lahaye, et al., Phys. Rev. Lett. 101, 080401 (2008).

[36] In situ imaging may be possible in on-going Dy experiments [A. Griesmaier, (private communication)].

[37] The local spectrum should also play an important role in the stability spectroscopy of rotons, recently proposed by J. P. Corson, R. M. Wilson, and J. L. Bohn, Phys. Rev. A 87, 051605 (2013). In particular, the collapse will be first induced at the center of the trap, where the susceptibility against density perturbations is the largest.

[38] Effects of the roton confinement on Bragg spectroscopy may be inferred from some results of Ref. 25]. In particular, the roton confinement leads to a marked uncertainty $\left(\propto 1 / l_{*}\right)$ in the roton momentum. 